\title{
A Robust Statistical Method for Brain Magnetic Resonance Image Segmentation
}

\author{
Bo Qin ${ }^{1}$, JingHua Wen ${ }^{2}$, and Ming Chen ${ }^{1}$ \\ ${ }^{1}$ Department of Automatic Control of Northwestern Polytechnical University, Xi' an, \\ 710072, P.R. China \\ qinbo_2000@163.com \\ 2 School of Medicine of Xi' an JiaoTong University, Xi' an, 710049, P.R. China
}

\begin{abstract}
In this paper, a robust statistical model-based brain MRI image segmentation method is presented. The MRI images are modeled by Gaussian mixture model. This method, based on the statistical model, approximately finds the maximum a posteriori estimation of the segmentation and estimates the model parameters from the image data. The proposed strategy for segmentation is based on the EM and FCM algorithm. The prior model parameters are estimated via EM algorithm. Then, in order to obtain a good segmentation and speed up the convergence rate, initial estimates of the parameters were done by FCM algorithm. The proposed image segmentation methods have been tested using phantom simulated MRI data. The experimental results show the proposed method is effective and robust.
\end{abstract}

\section{Introduction}

Automatic and robust brain tissue classification from magnetic resonance images (MRI) is of great importance for anatomical imaging in brain research. Segmentation brain images can be used in the three-dimensional visualization and quantitative analysis of brain morphometry and functional cortical structures. Segmentation of the brain MRI image into different tissues, such as the gray matter (GM), the white matter (WM), the cerebrospinal fluid (CSF).Now, brain segmentation methods can be categorized as manual methods and semi automated and automated methods. In the study of brain disorders, a large amount of data is necessary but in most clinical applications, the manual slice segmentation is the only method of choice and is time consuming. Even if experts do it, these types of segmentation stays subjective and show some intra and inter variability. Fully automatic, robust tissue classification is required for batch processing the data from large-scale, multi-site clinical trials or research projects.

The automatic segmentation of brain MR images, however, remains a persistent difficult problem. The main artifacts affecting brain MRI scans such as Intensity nonuniformity, and image Noise and Partial volume effect. Currently available methods for MR image segmentation can be categorized into Region-based and clusteringbased techniques[1]. Region-based techniques include the use of standard image 
processing techniques such as threshold-based, and mathematical morphology-based, and probability-based, and clustering-based, and prior knowledge-based and neural network-based techniques [2-8].

This paper aims to develop an algorithm for the automatic estimation of the statistics of the main tissues of the brain [the gray matter (GM), the white matter (WM), the cerebrospinal fluid (CSF)] from MRI images. These statistics can be used for segmenting the brain from its surrounding tissues for 3-D visualization or for a quantitative analysis of the different tissues. Segmentation of MR brain images was carried out on original images using the Gaussian mixture model models (GMMS)[9-10] and fuzzy c-means [2] techniques. The segmentation method presented in this work models the intensity distributions of MRI images as a mixture of Gaussians. The prior model parameters are estimated via EM algorithm [8]. Then, in order to obtain a good segmentation and speed up the convergence rate, initial estimates of the parameters were done by FCM algorithm. The performance of the algorithm is evaluated using phantom images. The experiments on simulated MR images prove that our algorithm is insensitive to noise and can more precisely segment brain MRI images into different tissues: the gray matter, the white matter and the cerebrospinal fluid.

\section{Image Model}

In this section, we derive a model for the tissues of the brain. For this purpose, we consider a normal human brain consists of three types of tissues: the white matter (WM), the gray matter (GM) and the cerebrospinal fluid (CSF). It is simplified in the case where only $\mathrm{T} 1$ weight images are considered. The image is defined by $y=\left(y_{i}, i \in I\right)$ where $y_{i}$ denotes the image intensity as the voxel indexed by $i$. We assume only one class of tissue occupies the spatial volume of each voxel. Let the total number of tissue classes in the image be $K$ and each of them be represented by a label from $\Lambda=\{1,2, \cdots K\}$ and $x_{i}$ represents the tissue class of voxel at the image site $i, x_{i}=k$ denote an assignment of the $k t h$ tissue class to the site $i$. A segmentation of the image is given by $x_{i}=\left(x_{i} ; i \in I\right)$. The process of segmentation is to find $x$, which represents the correct tissue class at each voxel of image $y$., our attempt was to find $x=x^{*}$ which represents optimal segmentation is given by:

$$
x^{*}=\arg \max _{x} p(x \mid y)
$$

From Baye's theorem, the posterior probability of segmentation $p(x \mid y)$ can be written as:

$$
p(x \mid y) \propto p(x, y)=p(y \mid x) p(x)
$$

where $p(y \mid x)$ is the conditional probability of the image $y$ given the segmentation $x$ and $p(x)$ is the prior density of $x$.our attempt is to find the maximum a posteriori(MAP)estimate by modeling $p(y \mid x)$ the measurement model. Each tissue class has a signature, or mean intensity and variance at a particular site. For each tissue 
class, a gaussian distribution is assumed and the entire image can be assumed as a Gaussian mixture density. A tissue can be modeled by a multivatiate Gaussian density with mean vector $\mu$ and covariance matrix $\Sigma$, i.e.;

$$
p(x \mid \theta)=(2 \pi)^{-M / 2} \Sigma_{k}^{-1 / 2} \cdot \exp \left(-\frac{1}{2}\left(x_{i}-\mu_{k}\right)^{t} \Sigma_{k}^{-1}\left(x_{i}-\mu_{i}\right)\right)
$$

Where $\theta=\left(\mu_{k}, \Sigma_{k}\right)$ is the vector of parameters associated with each type of tissue $k, \mu_{k}=\left(\mu_{k 1}, \mu_{k 2}, \cdots, \mu_{k M}\right)^{t}$ is the mean vector, and $\Sigma_{k}=E\left[\left(x_{i}-\mu_{k}\right)\left(x_{i}-\mu_{k}\right)^{\prime}\right]$ is the covariance matrix associated with class $k, 1 \leq k \leq c$ where $c$ is the number of classes. In our case, since the input is intensity at a given point $i$, the dimension is one, and the number of classes $K=3$ corresponding to the gray matter(GM), the white matter(WM) and the cerebrospibal fluid.(CSF).

\section{MR Image Segmentation Framework}

\subsection{Initial Parameter Estimation}

The choice of initial parameter is very important. The initial classification can be obtained either directly through the thresholding or through ML estimation with those known parameters .In this work; we use a modified FCM algorithm [11] for initial classification. The modified fuzzy c-means (FCM) algorithm is the best known and the most widely used fuzzy clustering technique. This algorithm iteratively minimizes the following objective function:

$$
J=\sum_{i=1}^{C} \sum_{j=1}^{N}\left(u_{i j}\right)^{m} d^{2}\left(x_{j}, c_{i}\right)-a \sum_{i=1}^{C} p_{i} \log \left(p_{i}\right)
$$

Where $u_{i j}$ is the membership value at pixel $j$ in the class $i$ such that $\sum_{i=1}^{C} u_{i j}=1, \forall j \in[0, N] \cdot p_{i}=\frac{1}{N} \sum_{j=1}^{N} u_{i j}$ is interpreted as "probability" of all the pixels. $d^{2}\left(x_{j}, c_{i}\right)$ is the standard Euclidian distance and the fuzziness index $m$ is a weighting coefficient on each fuzzy membership.

\subsection{Parameters Estimation}

Now that we have defined a model for our data, the problem is to estimate the different parameters of the mixture. The aim of estimation is find the parameters that maximize the likelihood of the GMM, given the image $Y=\left\{y_{1}, y_{2}, \cdots, y_{T}\right\}$ the GMM likelihood can be written as

$$
p(Y \mid \theta)=\prod_{i=1}^{T} p\left(y_{i} \mid \theta\right)
$$


if $p(Y \mid \theta)$ is a well behaved, differentiable of $\theta$, then $\theta$ can be found by the standard methods of differential calculus. This expression is a nonlinear function of the parameters $\theta$ and direct maximization is not possible. However, ML parameter estimates can be obtained iteratively using a special case of the expectation-maximization (EM) algorithm.

The EM algorithm estimates the maximum likelihood parameter $\theta$,we seek:

$$
\hat{\theta}=\arg \max _{\theta} \log p(y \mid \theta)
$$

The EM algorithm is an iterative procedure for finding the ML estimate of the parameters. Each iteration consists of two steps:

$$
\begin{aligned}
& \text { E-Step: Find } Q\left(\theta \mid \theta^{(t)}\right)=E\left[\log f(x, \theta) \mid y, \theta^{(t)}\right] \\
& \text { M-Step:Find } \theta^{(t+1)}=\arg \max _{\theta}\left\{Q\left(\theta, \theta^{(t)}\right)\right\}
\end{aligned}
$$

The EM algorithm begins with an initial model $\theta$, and estimates a new model $\hat{\theta}$, such that $p(Y \mid \hat{\theta}) \geq p(Y \mid \theta)$. The new model then becomes the initial model for the next iteration and the process is repeated until some convergence threshold is reached .In the case of the univariate normal mixture, the maximum likelihood estimates $\hat{w}_{i}$ of the mixture coefficients, $\hat{\mu}_{i}$ of the mean and $\Sigma_{i}$ of the variance are expressed as fllows:

$$
\begin{gathered}
\hat{w}_{i j}=\frac{a_{i} p\left(y_{i} \mid \mu_{i}, \sum_{i}\right)}{\sum_{l=1}^{K} a_{l} p\left(y_{j} \mid \mu_{l}, \Sigma_{l}\right)} \\
\hat{a}_{i}=\frac{1}{N} \sum_{i=1}^{N} w_{i j}, \quad i=1,2, \ldots, K \\
\hat{\mu}_{i}=\frac{\sum_{j=1}^{N} w_{i j} y_{j}}{\sum_{j=1}^{N} w_{i j}}, \quad i=1,2, \ldots, K \\
\sum_{i}=\frac{w_{i j}\left(y_{j}-\mu_{i}\right)\left(y_{j}-\mu_{i}\right)^{\prime}}{\sum_{j=1}^{N} w_{i j}}, \quad i=1,2, \ldots, K
\end{gathered}
$$

In our work, as mentioned earlier a three-tissue gaussian model was assumed to characterize the gray matter, the white matter and the cerebrospinal fluid. The EM algorithm was used to estimate the parameters of the gaussian mixture. Improved segmentation resulted when images were used. The convergence of EM algorithm was faster when initial estimates of the parameters were done by Fuzzy c- means. 


\section{Experimental Results}

In this section we describe the performance of our method to the segmentation of the brain into white matter and gray matter and CSF. To validate the performance of our method, we use the Brainweb MRI simulator(http://www.bic.mni.mcgill.ca/ brainweb), which consists of 3-dimensional MR data simulated using TI weight image, each data set is composed of voxels of $181 \mathrm{X} 217 \mathrm{X} 181$, the slice thickness is $1 \mathrm{~mm}$. The 2-D images are slice from the 3-D data sets.2-D segmentation is the clustering of the slice images,3-D segmentation is the clustering of the whole 3-D data sets. We use several simulated MRI acquisitions of this phantom including RF non-uniformities and noise levels. Segment has been done on MR images containing 3, 5 and $9 \%$ noise and of $20 \%$ RF non-uniformity. The brain data were classified into three clusters: gray matter, white matter and cerebrospinal fluid. Fig.1-

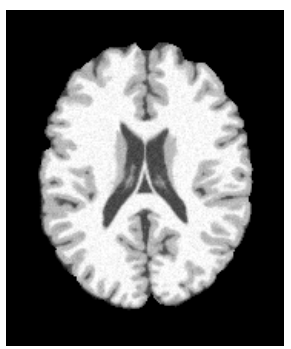

(a)

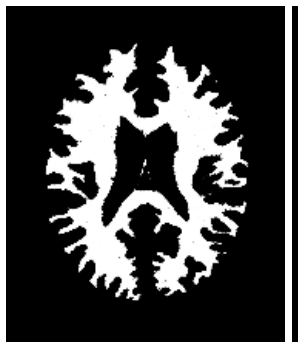

(b)

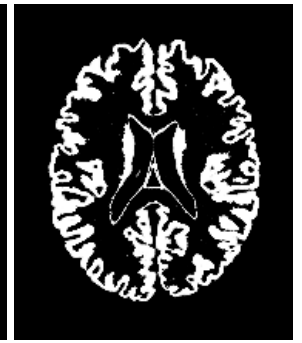

(c)

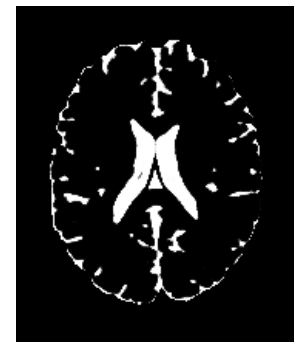

(d)

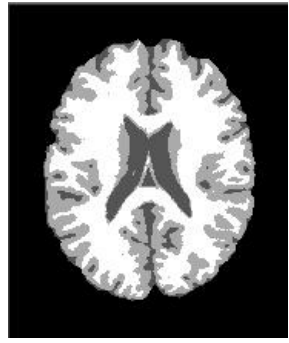

(e)

Fig. 1. Segmentation Result under $3 \%$ noise (a) T1 weight image (b)-(d) GM, WM and CSF posterior functions computed by our method respectively (e) segmentation result

Fig.3 show the segmentation results on the simulated MRI images with different noise level. Although the images with $9 \%, 5 \%$ noise look much worse than the images with $3 \%$ noise, there is noticeable difference on the segmentation images by the proposed method as shown in Fig.2-Fig.3.Fig.4 shows the final 3D rendering of the gray matter and white matter volume using the proposed segmentation method. 


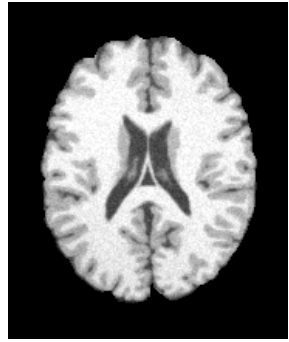

(a)

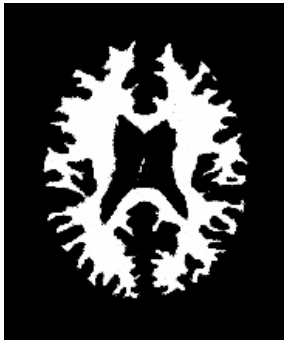

(b)

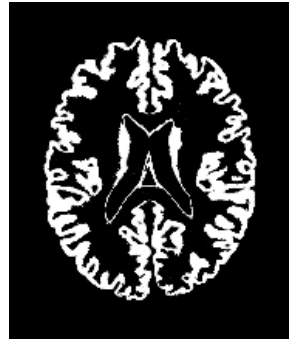

(c)

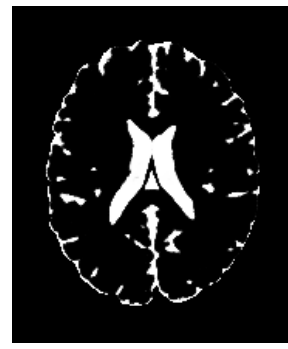

(d)

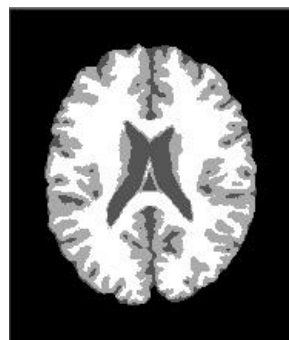

(e)

Fig. 2. Segmentation Result under 5\% noise: (a) T1 weight image (b)-(d) GM, WM and CSF posterior functions computed by our method respectively (e) segmentation result

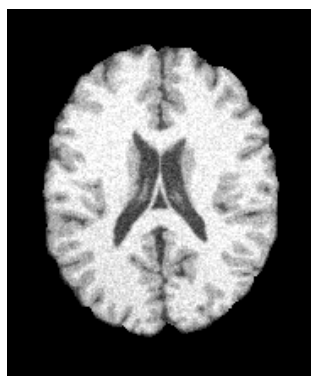

(a)

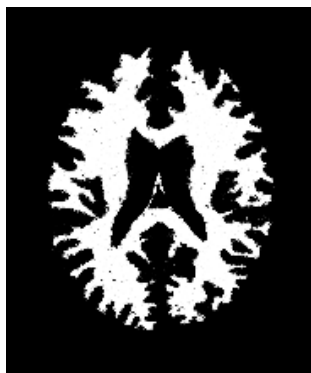

(b)

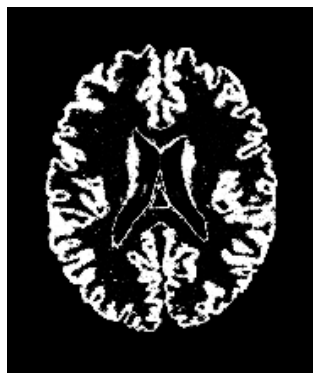

(c)

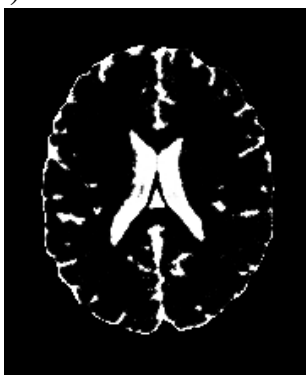

(d)

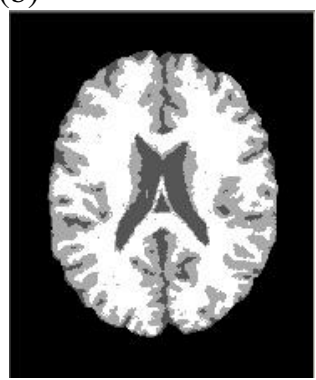

(e)

Fig. 3. Segmentation Result under 9\% noise: (a) T1 weight image (b)-(d) GM, WM and CSF posterior functions computed by our method respectively (e) segmentation result 

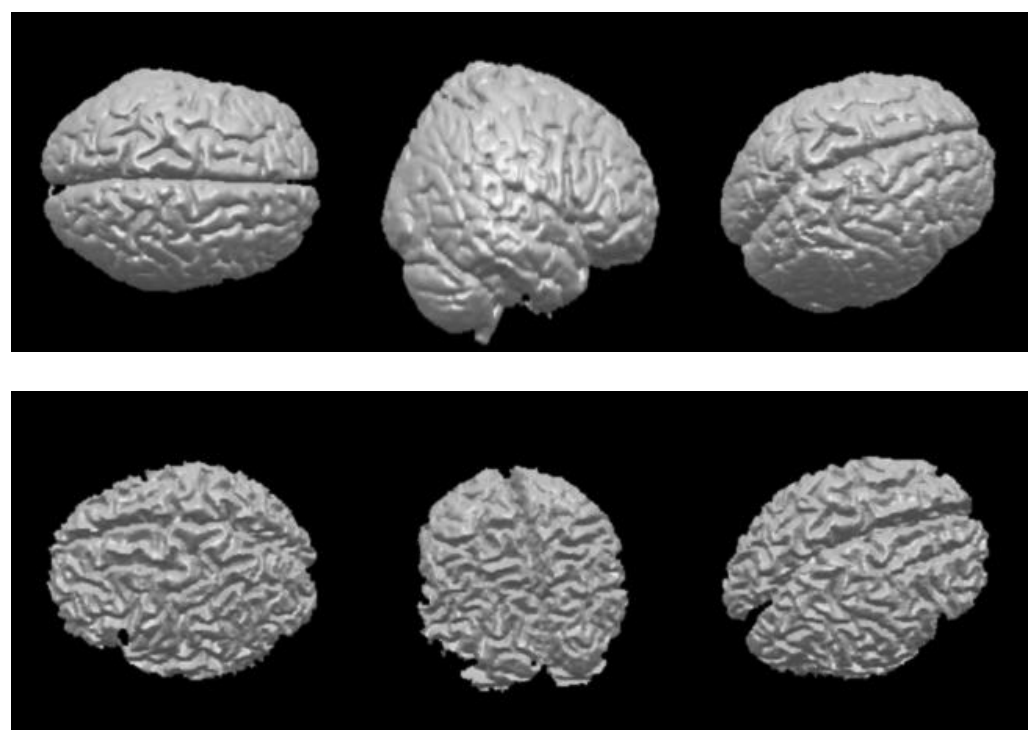

Fig. 4. 3D rendering of the gray matter and white matter

\section{Conclusions}

We have presented an approach combining Gaussian mixture model finite mixture model and FCM clustering algorithm. The parameters initialization using Fuzzy cmeans algorithms. The proposed image segmentation methods have been tested using phantom simulated MRI data and real MRI brain data. The experiments on simulated MR T1-Weight brain images prove that our algorithm is insensitive to noise and can more precisely segment brain MRI images into different tissues: gray matter, white matter and cerebrospinal fluid.

\section{References}

[1] Pham, D.L., Xu, C., Prince, J.L.: Current methods in medical image segmentation.Annual Review of Biomedical Engineering,Vol. 2, pp.315-337,2000.

[2] M.C.Clark,L.O.Hall, and D.B,Goldgof, MRI segmentation using fuzzy clustering techniques: integrating knowledge, IEEE Eng Med Biol;Vol.13(5),pp.730-742, 1994.

[3] M.Ozkan, and B. M. Dawant, Neural-Network Based Segmentation of Multi-Modal Medical Images, IEEE Transaction on Medical Imaging, Vol.12, pp.534-544, 1993.

[4] Kapur, T., Grimson, W.E., Wells, W.M., Kikinis, R., Segmentation of brain tissue from magnetic resonance images. Med Image Anal. Vol. 1 pp.109-127, 1996.

[5] Y.Wang,and T.Adali, Quantification and segmentation of brain tissues from MR images: A probabilistic neural network approach, IEEE Trans. on Image Processing, Vol.7, pp.1165-1180, 1998.

[6] C.Tsai, BS.Manjunath, and R.Jagadeesan, Automated segmentation of brain MR images", Pattern Recogn, Vol.28, pp.1825-1862, 1995. 
[7] W. M. Wells, and W. E. L. Grimson, Adaptive Segmentation of MRI data, IEEE Transaction on Medical Imaging, Vol.15, pp.429-442, 1996.

[8] Leemput, K.V., Maes, F., Vandermeulen, D., Suetens, P, Automated model-based tissue classi ${ }^{-}$cation of MR images of the brain. IEEE trans. on medical imaging,Vol.18, pp.897908, 1999.

[9] G. J. McLachlan, and T. Krishnan. The EM algorithm and extensions. John Wiley and Sons, New York, 1996.

[10] G. M. McLachlan and D. Peel, Finite Mixture Models. New York: John Wiley \& Sons, Inc., 2001.

[11] A.Lorette, X.Descombes, and J.Zerubia, Urban aereas extraction based on texture analysis through a markovian modeling, International journal of computer vision Vol.36, pp,219234,2000 . 